\title{
Radiofrequency Ablation of Atrioventricular Nodal Reentrant Tachycardia in Patient with Budd-Chiari Syndrome
}

\author{
Peller Michal, Lodziński Piotr*, Gawalko Monika, Balsam Pawel and Glowczynska Renata \\ Department of Cardiology, Medical University of Warsaw, Poland
}

Received: June 18, 2018; Published: July 05, 2018

*Corresponding author: Lodziński Piotr, $1^{\text {st }}$ Chair and Department of Cardiology, Medical University of Warsaw, ul. Banacha 1a, 02-097 Warszawa Poland

\section{Abstract}

Budd-Chiari syndrome is a rare disorder induced by thrombotic or non-thrombotic obstruction of the hepatic veins. We report a patient who underwent successful catheter ablation of atrioventricular nodal reentrant tachycardia (AVNRT) despite of inferior vena cava stenosis and heart displacement due to hepatomegaly. For better visualization of venous system 3D printed model of vena cava inferior was made (Figure 1).

\section{Introduction}

Budd-Chiari syndrome is a rare disorder caused by blood clots that completely or partially block blood flow from the liver. The blockage may occur anywhere, from hepatic veins to the inferior vena cava causing their occlusion. It may also result in displacement of anatomical heart position due to enlargement of the liver. We report a patient who underwent successful catheter ablation of atrioventricular nodal reentrant tachycardia (AVNRT) in spite of the mentioned unfavorable conditions [1].

\section{Case Report}

A 50-year-old woman with the diagnosis of end-stage liver disease due to Budd-Chiari syndrome was referred to our hospital for assessment prior liver transplantation. She was admitted with refractory ascites, esophageal varices and iron deficiency anemia.
The computer tomography of the abdomen shown in Figure 1A, revealed liver enlargement, considerable hypertrophy of caudate lobe with its veins and many collaterals. The inferior vena cava showed narrowing near the opening of the hepatic veins. Portal vein flow was visualized, and the hepatic veins were invisible. The workup for the etiology of Budd-Chiari syndrome, including blood tests demonstrate mutation in JAK2 V617F gene. Due to the frequent episodes of rapid palpitation caused by supraventricular tachycardia (first documented 4 months ago), further diagnostic tests were performed. During the palpitations, the electrocardiogram showed regular narrow complex tachycardia at 155 beats per minute (Figure 1B). Reduced blood pressure (85/40 $\mathrm{mmHg}$ ) was noticed during arrhythmia. Resting echocardiography detected no abnormalities of left ventricular (LV) function with ejection fraction of $60 \%$ [2].

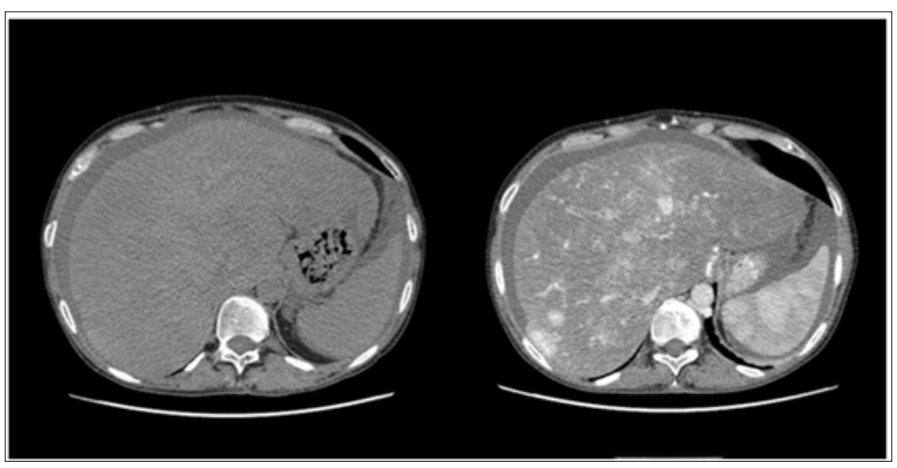

Figure 1A: Unenhanced (A), contrast-enhanced transverse CT scan (B). 


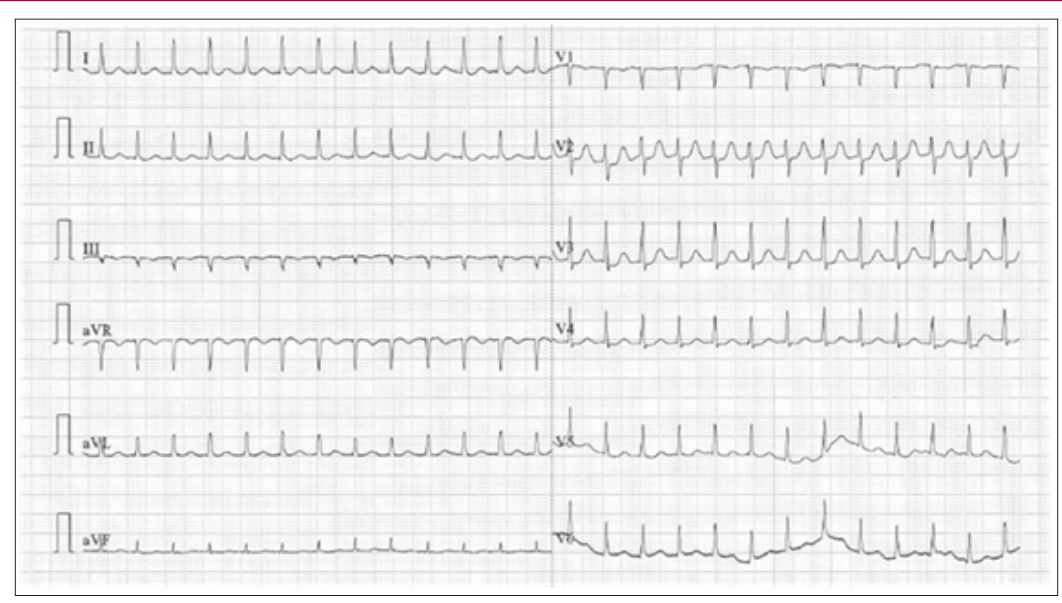

Figure 1B: ECG with atrioventricular nodal reentrant tachycardia.

During Holter ECG monitoring AVNRT was noted and lasted for 11 hours. Because of hemodynamically unstable arrhythmia and the risk of more pronounced drop off blood pressure during liver transplantation, patient was referred for ablation. Before the procedure, for better visualization of venous system 3D printed model of vena cava inferior was made (Figure 1C). The diagnosis of AVNRT was confirmed during the electrophysiologic study and successfully treated with radiofrequency catheter ablation (Figure
1D). There were no difficulties with femoral vein and inferior vena cava approach, despite a significant stenosis. However, hepatomegaly significantly changed anatomical position of the heart, which increased difficulty of the procedure. Two days after the procedure patient was discharged from the hospital. For the next one year of follow-up, there was no recurrence of arrhythmia, even during successful and uneventful procedure of liver transplantation [3].

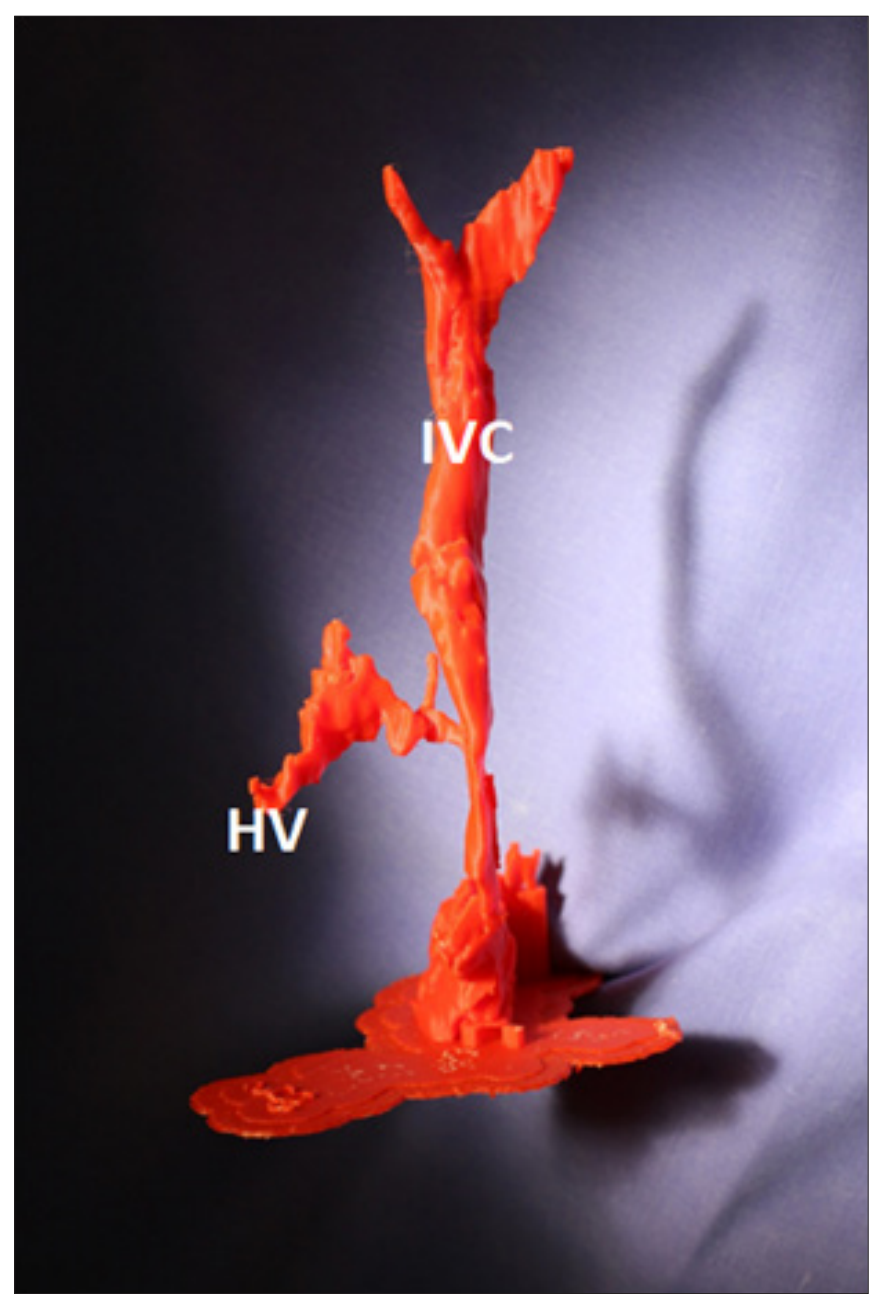

Figure 1C: 3D printing of inferior vena cava. Inferior vena cava (IVC) stenosis in the area of ostium of remaining patent hepatic vein (HV). 


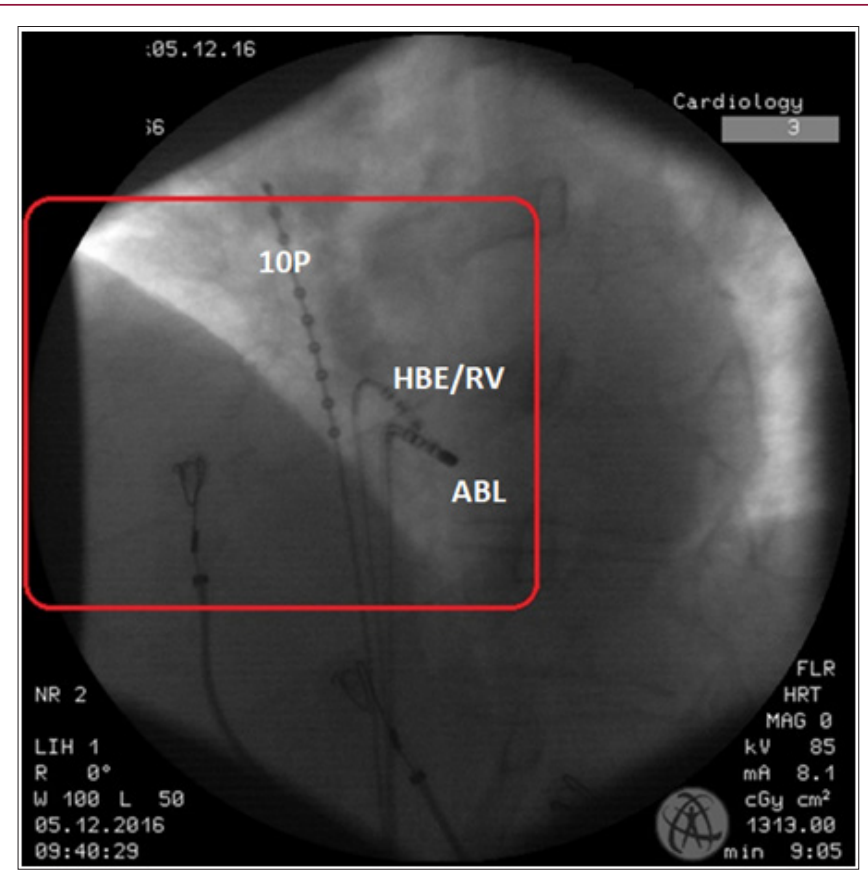

Figure 1D: Position of the catheters. In the right side of the picture (red box) enlarged liver isseen. (10P - 10-pole catheter in right atrium; HBE/ RV - catheter in the area of bundle of His and right ventricle; ABL - ablation catheter in the area of slow pathway).

\section{Conclusion}

Based on the above case, catheter ablation of the slow AV nodal pathway can be performed safely and successfully by inferior vena cava approach despite a significant stenosis and anatomical inconveniences in patients with Budd-Chiari syndrome.

\section{References}

1. Karthigesan M, Jayaprakash S (2009) Radiofrequency Catheter Ablation of Atrioventricular Nodal Reentry Tachycardia in A Patient with Inferior Vena Cava Anomaly. Indian Pacing Electrophysiol J 9(4): 214-218.

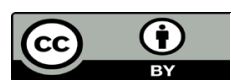

This work is licensed under Creative Commons Attribution 4.0 License

Submission Link: https://biomedres.us/submit-manuscript.php
2. Menon KV, Shah V, Kamath PS (2004) The Budd-Chiari syndrome. N Engl J Med 350: 578-585.

3. Jackman WM, Beckman KJ, McClelland JH, Xunzhang Wang, Karen J Friday, et al. (1992) Treatment of supraventricular tachycardia due to atrioventricular nodal reentry, by radiofrequency catheter ablation of slow pathway conduction. N Engl J Med 327: 313-318.

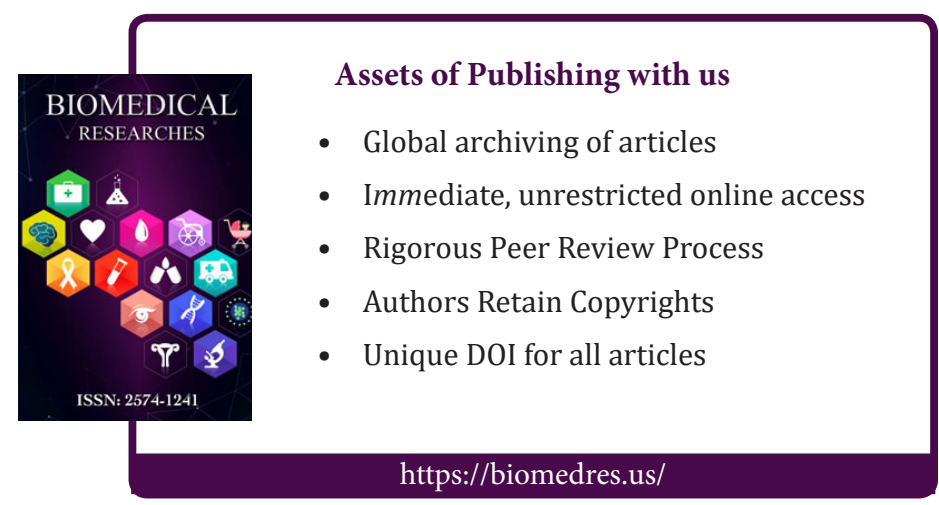

